QUARTERLY OF APPLIED MATHEMATICS

VOLUME LXIV, NUMBER 3

SEPTEMBER 2006, PAGES 447-461

S $0033-569 X(06) 01031-6$

Article electronically published on August 15, 2006

\title{
HOMOCLINIC ORBITS IN PREDATOR-PREY SYSTEMS WITH A NONSMOOTH PREY GROWTH RATE
}

\author{
BY \\ JITSURO SUGIE (Department of Mathematics and Computer Science, Shimane University, \\ Matsue 690-8504, Japan) \\ AND \\ KYOKO KIMOTO (Department of Mathematics and Computer Science, Shimane University, \\ Matsue 690-8504, Japan) \\ Dedicated to Professor Tadayuki Hara on the occasion of his 60th birthday
}

Abstract. This paper deals with Gause-type predator-prey models with a nonsmooth prey growth rate. Our models have a unique positive equilibrium and are under the influence of an Allee effect. A necessary and sufficient condition is given for the existence of homoclinic orbits whose $\alpha$ - and $\omega$-limit sets are the positive equilibrium. The argument used here is based on some results of a system of Liénard type. The relation between homoclinic orbits and the Allee effect is clarified. A simple example is included to illustrate the main result. Some global phase portraits are also attached.

1. Introduction. A class of Gause-type predator-prey systems is one of the most important mathematical models in population ecology. The following is a typical form of this model:

$$
u^{\prime}=u r(u)-p(u) v, \quad v^{\prime}=v(q(u)-D) .
$$

Here $u(t)$ and $v(t)$ are prey and predator densities, respectively; ' $=d / d t ; p(u)$ is the capture rate of prey per predator (the rate is called a functional response of predators to prey); $q(u)$ is the growth rate of the predator enhanced by an amount proportional to the prey density; $r(u)$ is the density-dependent growth rate of the prey in the absence of any predators; $D>0$ is the death rate of the predator in the absence of any preys (for details, see [3, Chapter 4]).

Received July 20, 2005.

2000 Mathematics Subject Classification. Primary 34C37, 37N25, 70K44; Secondary 34C05, 34D23, 92D25.

Key words and phrases. Gause-type predator-prey system, Allee effect, homoclinic orbits, global asymptotic stability, Liénard system.

The first author was supported in part by Grant-in-Aid for Scientific Research 16540152.

E-mail address: jsugie@riko.shimane-u.ac.jp 
System (1.1) includes various kinds of famous models (say, Lotka-Volterra type, Holling type, Ivlev type) as specific cases. It naturally has been studied by a number of authors; for example, results can be found in [1, 2, 3, 4, 5, 6, 7, 8, 9, 10, 11, 12, 13, 14, 15, 16, 17, 20, 21, 22 and the references cited therein. In the literature it is assumed that the rates $r(u), q(u)$ and $p(u)$ are sufficiently smooth on $[0, \infty)$ and satisfy

(i) $r(0)>0$ and there exists a $K>0$ such that $r(K)=0$ and $(u-K) r(u)<0$ for $u \neq K$

(ii) $q(0)=0, \frac{d}{d u} q(u)>0$ for all $u>0$ and there exists a $u^{*} \in(0, K)$ such that $q\left(u^{*}\right)=D$

(iii) $p(0)=0$ and $\frac{d}{d u} p(u)>0$ for all $u>0$, respectively. Let $v^{*}=u^{*} r\left(u^{*}\right) / p\left(u^{*}\right)$. Then, from the assumptions above, the point $E^{*}\left(u^{*}, v^{*}\right)$ is a unique equilibrium in the first quadrant $Q=\{(u, v): u>0$ and $v>0\}$. We call $E^{*}$ the positive equilibrium hereafter.

The main subjects of system (1.1) are the existence of a unique limit cycle which surrounds the positive equilibrium $E^{*}$ (see [1, 9, 10, 11, 13, 16, 17, 21, 22]) and the global asymptotic stability of the positive equilibrium $E^{*}$ (refer to [2, 6, 12, 20]). The positive equilibrium $E^{*}$ is said to be globally asymptotically stable if $E^{*}$ is stable and if every orbit tends to $E^{*}$.

It is easy to show that all solutions of (1.1) are bounded in the future and remain in the first quadrant $Q$. Hence, the Poincaré-Bendixson theorem shows that if system (1.1) has a unique limit cycle, then $E^{*}$ has to be unstable. Indeed, $E^{*}$ is locally repulsive; that is, there exists some neighborhood $U$ of $E^{*}$ such that each orbit of (1.1) starting at every point in $U$ goes away from $U$ and each orbit of (1.1) starting at every point in $Q \backslash U$ does not enter into $U$. The smoothness of the rates $r(u), q(u)$ and $p(u)$ in system (1.1) plays a major role in showing that $E^{*}$ is locally repulsive when system (1.1) has a unique limit cycle.

In a general dynamical system, however, an equilibrium is not always locally repulsive even if it is unstable. An orbit is said to be homoclinic if its $\alpha$ - and $\omega$-limit sets are the same equilibrium. If a homoclinic orbit exists, then the corresponding equilibrium is unstable, but it is not locally repulsive.

Sugie and Katayama [20] have shown that under the above assumptions (i)-(iii) and the smoothness conditions on $r(u), q(u)$ and $p(u)$, system (1.1) has no homoclinic orbits. From their result we see that there are only two possibilities: either system (1.1) has at least one limit cycle or the positive equilibrium $E^{*}$ is globally asymptotically stable. It is safe to say that system (1.1) has relatively simple global phase portraits because of the smooth rates.

A question naturally arises as to what will happen in the case of nonsmooth rates. Does a homoclinic orbit appear in system (1.1) for lack of smoothness of the rates? To give an answer to this question, we consider the predator-prey system with a nonsmooth prey growth rate:

$$
u^{\prime}=u(\beta-\gamma|u-\alpha|)-u v, \quad v^{\prime}=v\left(u^{2}-\alpha^{2}\right),
$$


where $\alpha, \beta$ and $\gamma$ are positive constants. System (1.2) has the positive equilibrium $E^{*}$ at $\left(u^{*}, v^{*}\right)=(\alpha, \beta)$. Since

$$
p(u)=u, \quad q(u)=u^{2}, \quad r(u)=\beta-\gamma|u-\alpha| \quad \text { and } \quad D=\alpha^{2}
$$

in system (1.2), assumption (ii) holds and assumption (iii) is satisfied with $u^{*}=\alpha$. Also, assumption (i) is satisfied with $K=\alpha+\beta / \gamma$ if $\beta>\alpha \gamma$. Otherwise $r(u)$ does not satisfy the assumption (i). Note that $r(u)$ is continuous for all $u \geq 0$, but is not differentiable at $u=\alpha$.

Our main result is stated as follows:

Theorem 1.1. System (1.2) has homoclinic orbits if and only if $0<\beta \leq \gamma^{2} / 8$.

When any predators are absent, system (1.2) is reduced to

$$
u^{\prime}=u(\beta-\gamma|u-\alpha|) .
$$

In this model, the growth rate of the prey increases with the prey density up to the peak $\beta$ and then the rate decreases as the density increases. Hence, equation (1.3) exhibits the so-called "Allee effect". In the case that $\beta<\alpha \gamma$, a low density leads to extinction. To be precise, if the density is lower than $\alpha-\beta / \gamma$, then the prey is strictly decreasing and will die out. The Allee effect is an important phenomenon in population dynamics.

From the above-mentioned relation between the prey growth rate and its density, we may say that system (1.2) is an ecological significant model simulating the effects of underpopulation and overpopulation of the prey. In system (1.2), the prey and the predator may coexist or they may die together. Only one side of the species cannot survive. For further details, see Section 4.

2. Proof of the main theorem. Consider a generalized Liénard system of the form

$$
\dot{x}=h(y)-F(x), \quad \dot{y}=-g(x),
$$

where ${ }^{\cdot}=d / d s ; F(x)$ and $g(x)$ are continuous on an open interval $I$ which contains 0 , and $h(y)$ is continuous and strictly increasing on $\mathbb{R}$. The functions $F(x), g(x)$ and $h(y)$ satisfy smoothness conditions for uniqueness of solutions of initial value problems. We assume that $F(0)=0$,

$$
x g(x)>0 \quad \text { if } x \neq 0
$$

and

$$
y h(y)>0 \text { if } y \neq 0 .
$$

Then the origin is the unique critical point of (2.1). Define the nonnegative function $G(x)$ by

$$
G(x)=\int_{0}^{x} g(\xi) d \xi
$$

Under the assumption that there exists an $m>0$ such that

$$
h(y) \leq m y
$$

for $y>0$ sufficiently small, Sugie 18 has presented the following sufficient conditions for system (2.1) to have homoclinic orbits (see also [19]). 
Theorem A. Assume (2.2) and suppose that

$$
F(x) \geq \sqrt{m}\{2 \sqrt{2 G(x)}-\phi(\sqrt{2 G(x)})\}
$$

for $|x|$ sufficiently small, where $\phi(\sigma)$ is a nonnegative continuous function satisfying

$$
\frac{\phi(\sigma)}{\sigma} \text { is nondecreasing }
$$

and there exists a constant $a>4$ such that

$$
a\left(\int_{0}^{\sigma} \frac{\phi(\xi)}{\xi^{2}} d \xi\right)^{2} \leq \frac{\phi(\sigma)}{\sigma}
$$

for $\sigma>0$ sufficiently small. Then system (2.1) has homoclinic orbits.

REMARK 2.1. We can easily find a nonnegative continuous function $\phi(u)$ satisfying conditions (2.4) and (2.5). For example, we may take $\phi(\sigma)=\sigma^{1+\varepsilon}$ for some $\varepsilon>0$; $\phi(\sigma)=b \sigma /(\log \sigma)^{2}$ with $0<b<1 / 4$.

Using Theorem A, we can prove the 'if'-part of Theorem 1.1.

Proof of the 'if'-part of Theorem 1.1. Changing variables

$$
x=u-\alpha, \quad y=\log \beta-\log v \quad \text { and } \quad d s=u d t,
$$

we can transform system (1.2) into system (2.1) with

$$
F(x)=\gamma|x|, \quad g(x)=x+\alpha-\frac{\alpha^{2}}{x+\alpha} \quad \text { and } \quad h(y)=\beta\left(1-e^{-y}\right),
$$

where $F(x)$ and $g(x)$ are defined on $(-\alpha, \infty)$, and $h(y)$ is defined on $\mathbb{R}$. Note that $F(0)=0, x g(x)>0$ if $x \neq 0$ and $y h(y)>0$ if $y \neq 0$. We get

$$
G(x)=\frac{1}{2} x^{2}+\alpha x-\alpha^{2} \log \left(\frac{x+\alpha}{\alpha}\right) .
$$

Let $m=\beta$. Then it is easy to check that

$$
h(y) \leq m y,
$$

namely, condition (2.2). We take $\phi(\sigma)=\sigma \sqrt{\sigma}$. Then it is clear that $\phi(\sigma)$ is nonnegative and continuous for $\sigma>0$. Since $\phi(\sigma) / \sigma=\sqrt{\sigma}$, condition (2.4) holds. We have

$$
a\left(\int_{0}^{\sigma} \frac{\phi(\xi)}{\xi^{2}} d \xi\right)^{2}=4 a \sigma<\sqrt{\sigma}=\frac{\phi(\sigma)}{\sigma}
$$

for $\sigma>0$ sufficiently small. Thus, condition (2.5) is also satisfied with arbitrary $a>4$.

We will show that condition (2.3) holds for $|x|$ sufficiently small. Since $\beta \leq \gamma^{2} / 8$, it is enough to show that

$$
F(x) \geq \frac{\gamma}{2}\{2 \sqrt{G(x)}-\sqrt{G(x)} \sqrt[4]{2 G(x)}\},
$$

namely,

$$
|x| \geq \sqrt{G(x)}\left\{1-\frac{1}{2} \sqrt[4]{2 G(x)}\right\}
$$


in some neighborhood of $x=0$. We first estimate that

$$
\begin{gathered}
g(x)<2 x \text { for } x>-\alpha \text { and } x \neq 0 \\
g(x)>3 x \text { for }-\frac{\alpha}{2}<x<0 .
\end{gathered}
$$

In fact, we have

$$
\frac{d}{d x} g(x)=1+\frac{\alpha^{2}}{(x+\alpha)^{2}} \quad \text { and } \quad \frac{d^{2}}{d x^{2}} g(x)=-\frac{2 \alpha^{2}}{(x+\alpha)^{3}}<0
$$

for $x>-\alpha$, and therefore,

$$
\left.\frac{d}{d x} g(x)\right|_{x=0}=2
$$

and $g(x)$ is a convex function. We also have

$$
g\left(-\frac{\alpha}{2}\right)=-\frac{3}{2} \alpha=3\left(-\frac{\alpha}{2}\right) .
$$

From (2.8) we see that

$$
\begin{gathered}
\sqrt{G(x)}<|x| \text { for } x>0 \\
|x|<\sqrt{G(x)}<\sqrt{\frac{3}{2}}|x| \text { for }-\frac{\alpha}{2}<x<0 .
\end{gathered}
$$

Hence, it is clear that (2.7) holds for $x \geq 0$. Let us examine whether (2.7) is also true for $x<0$ or not. To this end, we define

$$
f_{1}(x)=\frac{2}{\alpha} x^{2}-x+\alpha-\frac{\alpha^{2}}{x+\alpha} .
$$

Then, from a straightforward calculation, we conclude that there exists a $\delta>0$ such that $f_{1}(x)>0$ for $-\delta<x<0$; that is,

$$
\frac{1}{\alpha} x-1<\frac{g(x)}{-2 x}<0 \text { for }-\delta<x<0
$$

We may assume without loss of generality that $\delta<\min \left\{\alpha / 2, \alpha^{2} / 4\right\}$. Hence, together with (2.9), we obtain

$$
\frac{1}{\alpha} x-1<\frac{g(x)}{2 \sqrt{G(x)}}<0 \text { for }-\delta<x<0 .
$$

We next define

$$
f_{2}(x)=-x-\sqrt{G(x)}+\frac{1}{2} \sqrt{G(x)} \sqrt[4]{2 G(x)} .
$$

Then we have

$$
f_{2}(0)=0 \quad \text { and } \quad \frac{d}{d x} f_{2}(x)=-1-\frac{g(x)}{2 \sqrt{G(x)}}+\frac{3 g(x)}{8 \sqrt{G(x)}} \sqrt[4]{2 G(x)} .
$$


From (2.8)-2.10) and the fact that $\delta<\alpha^{2} / 4$, we see that

$$
\begin{aligned}
\frac{d}{d x} f_{2}(x) & <-1-\left(\frac{1}{\alpha} x-1\right)-\frac{3}{8} \frac{2}{\sqrt{3 / 2}} \sqrt[4]{2 G(x)} \\
& <\frac{1}{\alpha}|x|-\frac{\sqrt{6} \sqrt[4]{2}}{4} \sqrt{|x|}<\frac{1}{\alpha}|x|-\frac{1}{2} \sqrt{|x|}<0
\end{aligned}
$$

for $-\delta<x<0$. Hence, (2.7) holds for $-\delta<x<0$.

By means of Theorem A, we conclude that system (2.1) with (2.6) has homoclinic orbits, and so has system (1.1). The proof is complete.

To prove the 'only if'-part of Theorem 1.1, we need the following result, which is a slight modification of Theorem 2.5 in 19 .

Theorem B. Suppose that there exists an $m>0$ such that

$$
h(y) \geq m y
$$

for $y>0$ sufficiently small. Also, suppose that

$$
F(x) \leq \sqrt{m}\{2 \sqrt{2 G(x)}-\psi(\sqrt{2 G(x)})\}
$$

for $x>0$ or $x<0,|x|$ sufficiently small, where $\psi(\sigma)$ is a nonnegative continuous function satisfying

$$
\frac{\psi(\sigma)}{\sigma} \text { is nondecreasing and is not greater than } 2
$$

and there exists a $\sigma_{0}>0$ such that

$$
\int_{0}^{\sigma_{0}} \frac{\psi(\xi)}{\xi^{2}} d \xi=\infty .
$$

Then system (2.1) fails to have homoclinic orbits.

REMARK 2.2. As simple examples satisfying conditions (2.13) and (2.14), we can cite $\psi(\sigma)=\varepsilon \sigma$ with $\varepsilon>0 ; \psi(\sigma)=-\sigma / \log \sigma$ for $\sigma>0$ sufficiently small.

Proof of the 'only if'-part of Theorem 1.1. As in the proof of the 'if'-part, we see that system (1.1) is equivalent to system (2.1) with (2.6). We will show that if $\beta>\gamma^{2} / 8$, then conditions (2.11) -2.14 in Theorem B are satisfied.

Since $\beta>\gamma^{2} / 8$, there exists a $\rho>0$ such that

$$
\frac{\gamma}{2}+\rho=\sqrt{2 \beta} .
$$

Let $\varepsilon$ be chosen so that $0<\varepsilon<\min \{1, \gamma / 2,2 \rho /(\gamma+4)\}$. Since

$$
\left.\frac{d}{d y} h(y)\right|_{y=0}=\beta,
$$

condition (2.11) is satisfied with $m=\beta-\varepsilon^{2} / 2$. Define $\psi(\sigma)=\varepsilon \sigma$. Then $\psi(\sigma)$ is nonnegative and continuous for $\sigma>0$ and satisfies conditions (2.13) and (2.14). We have only to show that condition (2.12) also holds. From the same manner as in the proof of the 'if'-part, we can estimate that

$$
|x|<\sqrt{G(x)} \text { for }-\alpha<x<0 .
$$


Hence, we have

$$
\begin{aligned}
\sqrt{m}\{2 \sqrt{2 G(x)}-\psi(\sqrt{2 G(x)})\} & =\sqrt{m}(2-\varepsilon) \sqrt{2 G(x)} \\
& =\sqrt{2 \beta-\varepsilon^{2}}(2-\varepsilon) \sqrt{G(x)} \\
& >(\sqrt{2 \beta}-\varepsilon)(2-\varepsilon) \sqrt{G(x)} \\
& >(\gamma / 2+\rho-\varepsilon)(2-\varepsilon)|x| \\
& >\{(2-\varepsilon) \rho-(\gamma / 2+2) \varepsilon+\gamma\}|x| \\
& >(\rho-\rho+\gamma)|x| \\
& =\gamma|x|=F(x)
\end{aligned}
$$

for $-\alpha<x<0$; namely, condition (2.12) is satisfied for $x<0,|x|$ sufficiently small.

Thus, by Theorem B we see that system (2.1) with (2.6) has no homoclinic orbits, and neither has system (1.1). This completes the proof.

REMARK 2.3. In the proof of the 'only if'-part above, we can also show that condition (2.12) is satisfied for $x>0$ sufficiently small. In fact, we can estimate that

$$
\sqrt{1-\varepsilon^{2}}|x|<\sqrt{G(x)}
$$

for $x>0$ sufficiently small, where $\varepsilon<\min \{1 / 3, \gamma / 2,2 \rho /(3 \gamma+4)\}$, and therefore, we have

$$
\begin{aligned}
\sqrt{m}\{2 \sqrt{2 G(x)}-\psi(\sqrt{2 G(x)})\} & >(\sqrt{2 \beta}-\varepsilon)(2-\varepsilon) \sqrt{G(x)} \\
& >(\gamma / 2+\rho-\varepsilon)(2-\varepsilon) \sqrt{1-\varepsilon^{2}}|x| \\
& >(\gamma / 2+\rho-\varepsilon)(2-\varepsilon)(1-\varepsilon)|x| \\
& >\{(2-\varepsilon)(1-\varepsilon) \rho-(3 \gamma / 2+2) \varepsilon+\gamma\}|x| \\
& >(\rho-\rho+\gamma)|x|=F(x)
\end{aligned}
$$

for $x>0$ sufficiently small.

3. Discussion. Let us return to the Gause model (1.1). Recall that system (1.1) has three density-dependent rates $p(u), q(u)$ and $r(u)$. We here suppose that $p(u)$ and $q(u)$ satisfy the assumptions (iii) and (ii) in Section 1, respectively; and $r(u)$ is continuous on $[0, \infty)$ with $r\left(u^{*}\right)>0$, where $u^{*}$ is a constant given in the assumption (ii). Note that this condition of $r(u)$ is easier than the assumption (i) in Section 1. Let $v^{*}=u^{*} r\left(u^{*}\right) / p\left(u^{*}\right)$. Then system (1.1) has a unique positive equilibrium $E^{*}\left(u^{*}, v^{*}\right)$.

By putting

$$
x=u-u^{*}, \quad y=\log v^{*}-\log v \quad \text { and } \quad d s=p(u) d t,
$$


system (1.1) is transformed into the system

$$
\dot{x}=\frac{\left(x+u^{*}\right) r\left(x+u^{*}\right)}{p\left(x+u^{*}\right)}-v^{*} e^{-y}, \quad \dot{y}=-\frac{q\left(x+u^{*}\right)-D}{p\left(x+u^{*}\right)} .
$$

Let

$$
F(x)=v^{*}-\frac{\left(x+u^{*}\right) r\left(x+u^{*}\right)}{p\left(x+u^{*}\right)} \quad \text { and } \quad g(x)=\frac{q\left(x+u^{*}\right)-D}{p\left(x+u^{*}\right)}
$$

for $x>-u^{*}$ and let

$$
h(y)=v^{*}\left(1-e^{-y}\right)
$$

for $y \in \mathbb{R}$. It is clear that $F(0)=0$ and $y h(y)>0$ if $y \neq 0$. From assumption (ii) we also see that $x g(x)>0$ for $x>-u^{*}$ and $x \neq 0$. Hence, system (3.1) is of Liénard type.

For the sake of simplicity, we denote

$$
\Gamma(x)=\frac{\left(x+u^{*}\right) r\left(x+u^{*}\right)}{\sqrt{v^{*}} p\left(x+u^{*}\right)}
$$

and

$$
\Delta(x)=\sqrt{2 \int_{0}^{x} \frac{q\left(\xi+u^{*}\right)-D}{p\left(\xi+u^{*}\right)} d \xi}
$$

for $x>-u^{*}$. Then, from Theorems $\mathrm{A}$ and $\mathrm{B}$, we obtain the following results.

Theorem 3.1. Suppose that

$$
\Gamma(0)-\Gamma(x) \geq 2 \Delta(x)-\phi(\Delta(x))
$$

for $|x|$ sufficiently small, where $\phi(\sigma)$ is a nonnegative continuous function satisfying conditions (2.4) and (2.5). Then system (3.1) has homoclinic orbits, and so has system (1.1).

Theorem 3.2. Suppose that there exists a constant $c$ with $0<c<1$ such that

$$
\Gamma(0)-\Gamma(x) \leq c\{2 \Delta(x)-\psi(\Delta(x))\}
$$

for $x>0$ or $x<0,|x|$ sufficiently small, where $\psi(\sigma)$ is a nonnegative continuous function satisfying conditions (2.13) and (2.14). Then system (3.1) does not have homoclinic orbits, and neither has system (1.1).

In single-species population dynamics, the equation

$$
u^{\prime}=\lambda u\left(1-\frac{u}{K}\right)
$$

is very famous as the logistic growth model. The parameters $\lambda$ and $K$ are positive, and they are called the intrinsic rate of increase and the carrying capacity for the prey density, respectively. The Allee effect is taken no account in equation (3.4).

As mentioned in Section 1, we can reduce system (1.2) to equation (1.3) in the case of absence of any predators. To contrast with system (1.2) and equation (1.3), we consider the system

$$
u^{\prime}=u(\beta-\gamma(u-\alpha))-u v, \quad v^{\prime}=v\left(u^{2}-\alpha^{2}\right)
$$

and the equation

$$
u^{\prime}=u(\beta-\gamma(u-\alpha)) .
$$


Arranging the right-hand side of (3.6), we see that this equation becomes the logistictype equation (3.4) with $\lambda=\beta+\alpha \gamma$ and $K=\alpha+\beta / \gamma$. System (3.5) naturally belongs to the Gause model (1.1). In the case of (3.5),

$$
\Gamma(x)=\sqrt{\beta}-\frac{\gamma}{\sqrt{\beta}} x \quad \text { and } \quad \Delta(x)=\sqrt{x^{2}+2 \alpha x-2 \alpha^{2} \log \left(\frac{x+\alpha}{\alpha}\right)} \geq 0
$$

for $x>-\alpha$, because $p(u)=u, q(u)=u^{2}, r(u)=\beta+\alpha \gamma-\gamma u, D=\alpha^{2}$ and $\left(u^{*}, v^{*}\right)=$ $(\alpha, \beta)$. Since

$$
\Gamma(0)-\Gamma(x)=\frac{\gamma}{\sqrt{\beta}} x
$$

for $x>-u^{*}$, it turns out that for $-\alpha<x<0$, conditions (2.13), (2.14) and (3.3) are satisfied with $c=1 / 2$ and $\psi(\sigma)=\sigma$, but condition (3.2) fails to hold for an arbitrary function $\phi(\sigma)$ satisfying conditions (2.4) and (2.5). Thus, by virtue of Theorem 3.2, we conclude that system (3.5) does not have homoclinic orbits. As a matter of fact, it is easy to prove that there exist no limit cycles in system (3.5). Hence, the positive equilibrium $E^{*}(\alpha, \beta)$ is always globally asymptotically stable for any parameters $\alpha, \beta$ and $\gamma$. But, to argue this point would carry us too far away from the purpose of this paper, and so we omit the details.

4. Numerical Example. To illustrate our results, we give an example and a series of figures. System (1.2) has three parameters. We fix the value of $\gamma$ at 2 and consider the system

$$
u^{\prime}=u(\beta-2|u-\alpha|)-u v, \quad v^{\prime}=v\left(u^{2}-\alpha^{2}\right),
$$

whose positive equilibrium $E^{*}$ is the point $(\alpha, \beta)$. The origin $E_{0}(0,0)$ and the point $E_{1}(\alpha+\beta / 2,0)$ are also equilibria of (4.1). We say that an equilibrium is a boundary equilibrium if it is on the $u$-axis.

In Figures 1-4, we classify some orbits in five types according to the following properties:

(i) an orbit starting at a point $P_{i}(i=1,2, \ldots, 6)$ rotates in a counterclockwise direction about $E^{*}$ infinitely many times and approaches $E^{*}$;

(ii) an orbit starting at a point $Q_{i}(i=1,2,3)$ tends to the origin as $t$ increases;

(iii) an orbit starting at a point $R_{i}(i=1,2, \ldots, 6)$ approaches $E^{*}$ without rotating about $E^{*}$ perpetually;

(iv) an orbit passing through a point $S_{i}(i=1,2, \ldots, 6)$ makes a loop together with $E^{*}$, namely, a homoclinic orbit;

(v) an orbit passing through a point $T_{i}(i=1,2,3)$ connects $E^{*}$ and the origin, in other words, its $\alpha$-limit set is $E^{*}$ and its $\omega$-limit set is the origin.

An orbit of type (v) is said to be heteroclinic. The broken line in each figure indicates the graph of $v=\beta-2|u-\alpha|$. For this reason, if an orbit meets the broken line, then it passes across the line in the vertical direction.

From Theorem 1.1 we see that system (4.1) has homoclinic orbits if and only if $0<\beta \leq$ $1 / 2$. In Figures $1-4$, we describe global phase portraits of (4.1) in the cases that $(\alpha, \beta)=$ $(1 / 8,3 / 4),(1 / 2,3 / 4),(1 / 8,1 / 3),(1 / 2,1 / 3)$, respectively. Hence, homoclinic orbits exist only in Figures 3 and 4. 
The first case: In Figure 1, we draw orbits of type (i). Each of them starts from one of the points $P_{1}(0.6,0.12), P_{2}(0.6,0.27), P_{3}(0.6,0.42), P_{4}(0.6,0.57), P_{5}(0.6,0.72)$ and $P_{6}(0.6,0.87)$. This figure shows that two boundary equilibria $E_{0}$ and $E_{1}$ are unstable and the positive equilibrium $E^{*}$ is globally asymptotically stable. This means that the prey and the predator always exist together.

The second case: As sketched in Figure 2, another boundary equilibrium appears at the point $E_{2}(\alpha-\beta / 2,0)$. The boundary equilibria $E_{1}$ and $E_{2}$ are unstable, and the origin $E_{0}$ and the positive equilibrium $E^{*}$ are locally asymptotically stable. There are orbits of two types (i) and (ii); each orbit starting at the point $P_{1}(1.2,0.08), P_{2}(1.2,0.18)$ or $P_{3}(1.2,0.28)$ approaches $E^{*}$ and each orbit starting at the point $Q_{1}(1.2,0.32), Q_{2}(1.2$, $0.42)$ or $Q_{3}(1.2,0.52)$ tends to $E_{0}$. Hence, $E^{*}$ is not globally asymptotically stable. Both species may become extinct.

The third case: There are nine orbits in Figure 3. Each orbit passing through the point $S_{1}(0.125,0.05), S_{2}(0.125,0.14)$ or $S_{3}(0.125,0.23)$ is homoclinic. The other orbits are not homoclinic, but they also tend to the positive equilibrium $E^{*}$. Each of them starts at the point $R_{1}(0.5,0.02), R_{2}(0.5,0.1), R_{3}(0.5,0.18), R_{4}(0.5,0.26), R_{5}(0.5,0.34)$ or $R_{6}(0.5,0.42)$. The boundary equilibria $E_{0}$ and $E_{1}$ are unstable. Since homoclinic orbits exist, needless to say, the positive equilibrium $E^{*}$ is not even stable. Although $E^{*}$ is unstable, it is globally attractive because every orbit approaches it. Hence, the prey coexists with the predator.

The fourth case: Figure 4 is more complicated than Figures 1-3. A remarkable feature of this case is the appearance of heteroclinic orbits joining the positive equilibrium $E^{*}$ and the boundary equilibrium $E_{0}$. Such heteroclinic orbits pass through one of the points $T_{1}(0.2,0.03), T_{2}(0.2,0.09)$ or $T_{3}(0.2,0.15)$. Each orbit passing through the point $S_{1}(0.5,0.05), S_{2}(0.5,0.15)$ or $S_{3}(0.5,0.25)$ is homoclinic. There are orbits of two types (ii) and (iii) besides heteroclinic orbits and homoclinic orbits; each orbit starting at the point $Q_{1}(1,0.21), Q_{2}(1,0.27)$ or $Q_{3}(1,0.33)$ tends to $E_{0}$ and each orbit starting at the point $R_{1}(1,0.02), R_{2}(1,0.08)$ or $R_{3}(1,0.14)$ approaches $E^{*}$. As in the second case, the boundary equilibria $E_{1}$ and $E_{2}$ are unstable. Since orbits of type (ii) or (v) appear, there is a high risk of extinction of the prey and the predator.

Finally, we direct our attention to a functional response of predators to prey. In system (1.2) or (3.5), the functional response $p(u)$ is $u$, which is strictly increasing. As shown in Figures 1-4, there exist no limit cycles in system (1.2) because of the monotonicity of the functional response. Holling [5] has proposed a bounded functional response from the factual datum. The functional response $u^{n} /\left(d+u^{n}\right)$ is said to belong to Holling type II if $0<n \leq 1$; to Holling type III if $n>1$. Holling-type functional responses are also strictly increasing, but are not unbounded. The functional response of Holling type II is upwards convex; that of Holling type III has an inflection point.

As is well known, there is a possibility that limit cycles appear in predator-prey models through the influence of a Holling-type functional response. For example, Sugie $e t$ al. [17, 20, 21] have considered system (1.1) with

$$
p(u)=\frac{u^{n}}{d+u^{n}}, \quad q(u)=\frac{\mu u^{n}}{d+u^{n}}, \quad \text { and } \quad r(u)=\lambda\left(1-\frac{u}{K}\right),
$$

and obtained a necessary and sufficient condition for the uniqueness of limit cycles. 


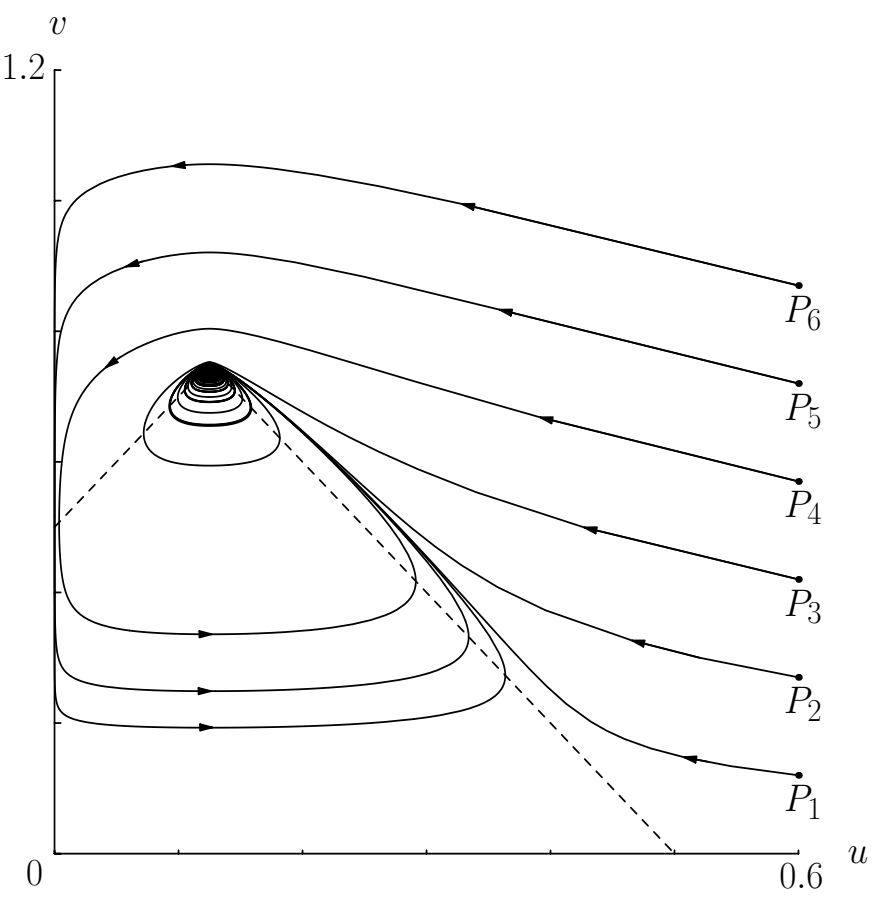

FIG. 1. Phase portrait for system (4.1) with $\alpha=1 / 8$ and $\beta=3 / 4$

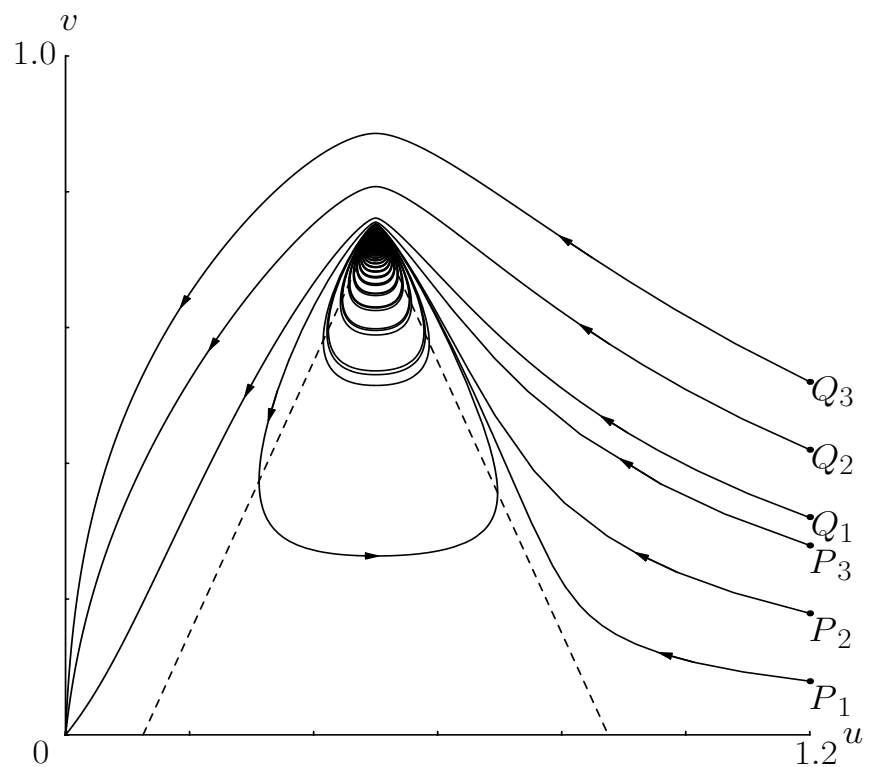

Fig. 2. Phase portrait for system (4.1) with $\alpha=1 / 2$ and $\beta=3 / 4$ 


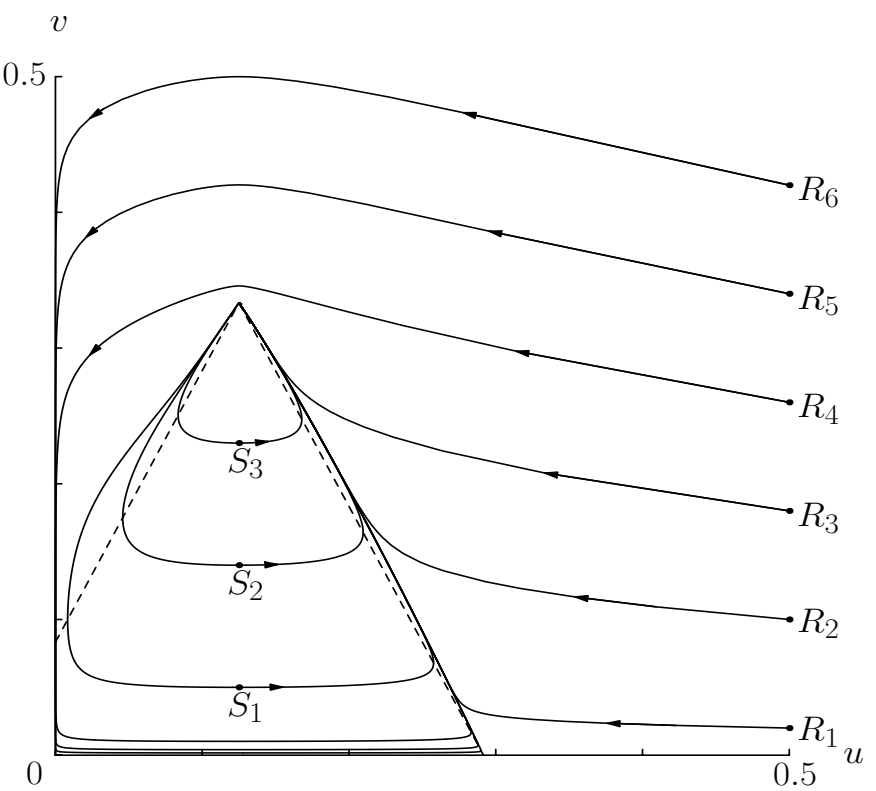

FIG. 3. Phase portrait for system (4.1) with $\alpha=1 / 8$ and $\beta=1 / 3$

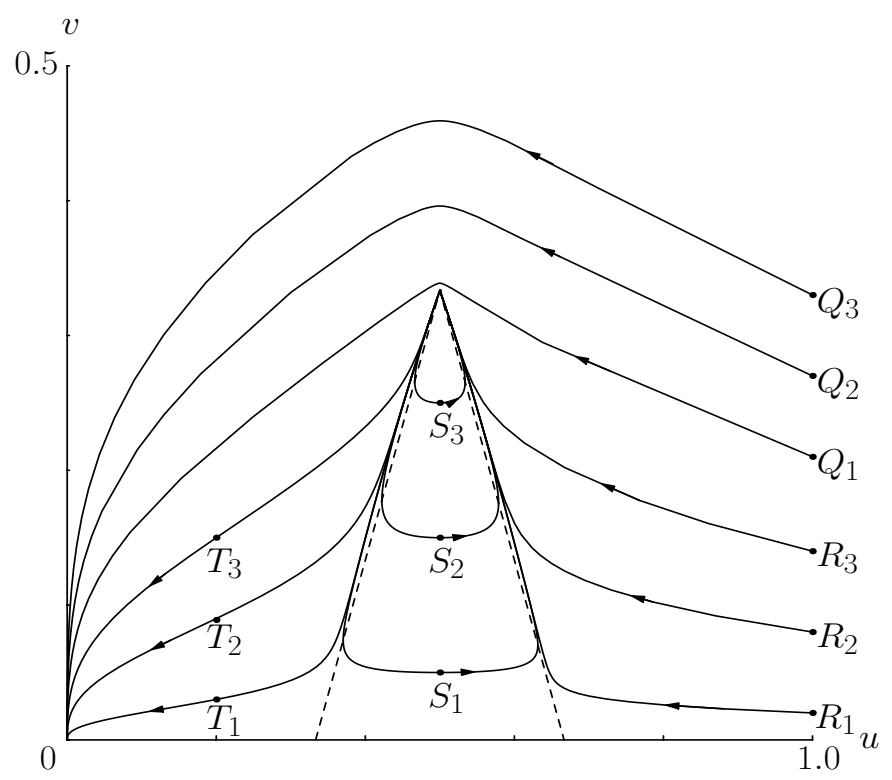

Fig. 4. Phase portrait for system (4.1) with $\alpha=1 / 2$ and $\beta=1 / 3$ 


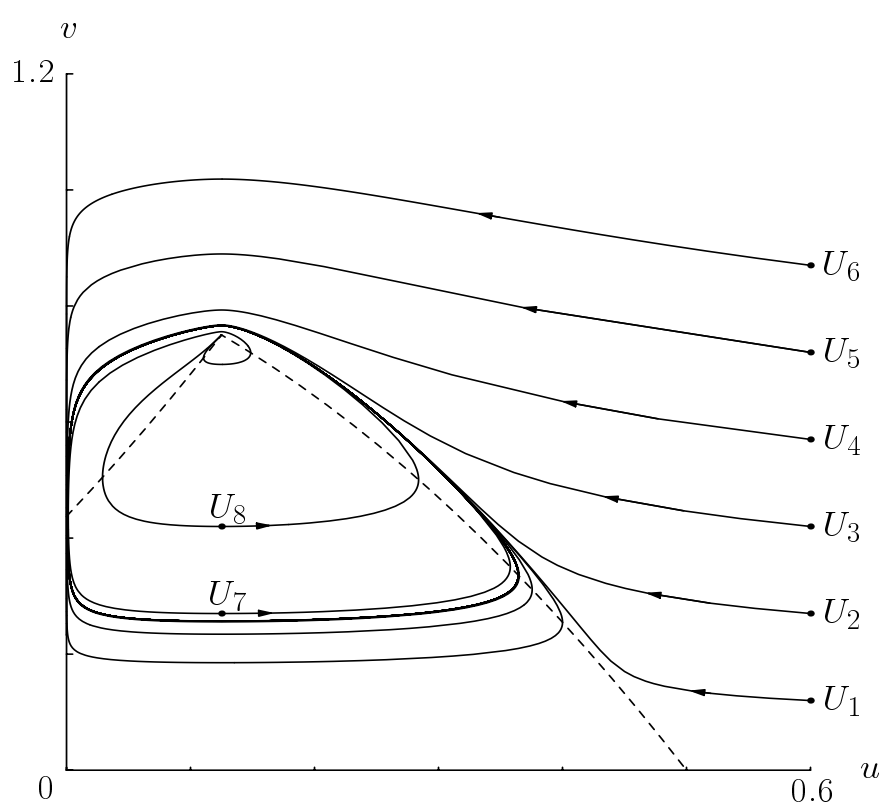

FIG. 5. Phase portrait for system (4.2) with $\alpha=1 / 8, \beta=3 / 4$ and $\gamma=2$

Let us consider the system

$$
u^{\prime}=u(\beta-\gamma|u-\alpha|)-\frac{u v}{1-\alpha+u}, \quad v^{\prime}=v\left\{\left(\frac{u}{1-\alpha+u}\right)^{2}-\alpha^{2}\right\}
$$

where $0<\alpha<1, \beta>0$ and $\gamma>0$, as a substitute for system (1.2). The functional response $u /(1-\alpha+u)$ of (4.2) belongs to Holling type II and the point $E^{*}(\alpha, \beta)$ is a unique positive equilibrium of (4.2). System (4.2) may have a limit cycle as well as homoclinic orbits.

If $\alpha=1 / 8, \beta=3 / 4$ and $\gamma=2$, then only one limit cycle exists. We describe eight orbits in Figure 5. Each orbit starts from one of the points $U_{1}(0.6,0.12), U_{2}(0.6,0.27)$, $U_{3}(0.6,0.42), U_{4}(0.6,0.57), U_{5}(0.6,0.72), U_{6}(0.6,0.87), U_{7}(0.125,0.27)$ and $U_{8}(0.125$, 0.42). All orbits rotate around the positive equilibrium $E^{*}$ clockwise and approach only one limit cycle. Hence, the limit cycle is globally asymptotically stable, and the equilibria $E^{*}, E_{0}$ and $E_{1}$ are unstable.

In case $\alpha=1 / 8, \beta=1 / 3$ and $\gamma=2$, homoclinic orbits appear (see Figure 6). The global phase portrait in this case looks very much like Figure 3 . There are orbits of two types (iii) and (iv); each orbit starting at the point $R_{1}(0.5,0.02), R_{2}(0.5,0.1)$, $R_{3}(0.5,0.18), R_{4}(0.5,0.26), R_{5}(0.5,0.34)$ or $R_{6}(0.5,0.42)$ tends to the positive equilibrium $E^{*}$ and each orbit passing through the point $S_{1}(0.125,0.05), S_{2}(0.125,0.14)$ or $S_{3}(0.125,0.23)$ is homoclinic. The equilibria $E^{*}, E_{0}$ and $E_{1}$ are unstable. 


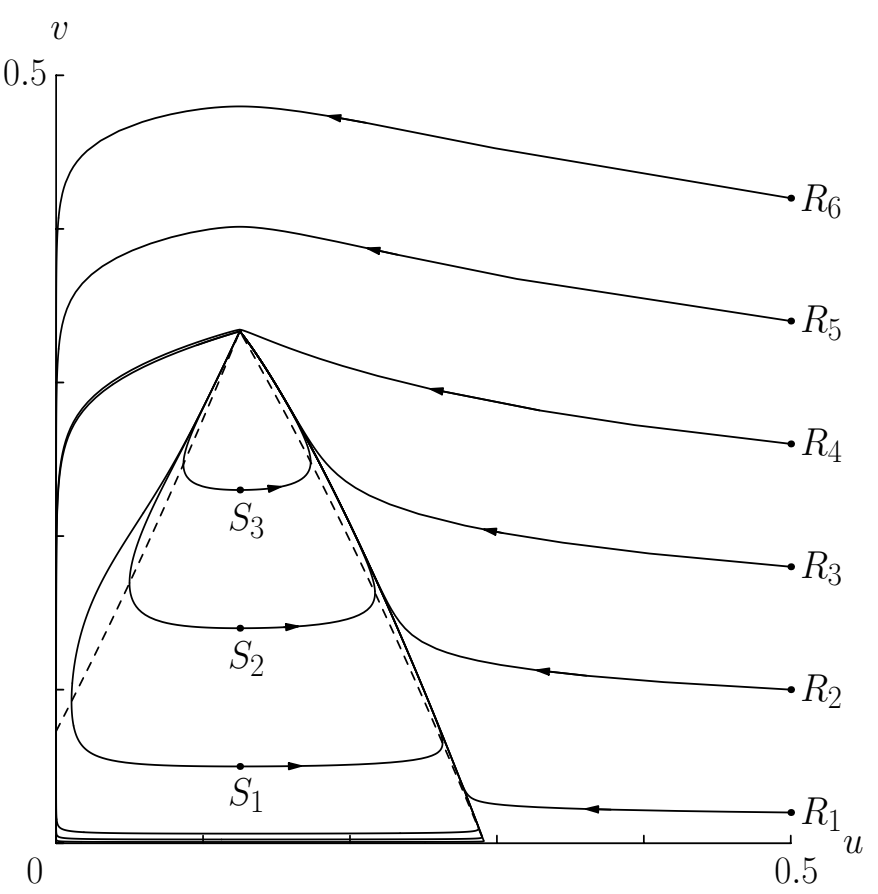

FIG. 6. Phase portrait for system (4.2) with $\alpha=1 / 8, \beta=1 / 3$ and $\gamma=2$

\section{REFERENCES}

[1] K.-S. Cheng, Uniqueness of a limit cycle for a predator-prey system, SIAM J. Math. Anal. 12, 541-548 (1981). MR0617713 (82h:34035)

[2] K.-S. Cheng, S.-B. Hsu and S.-S. Lin, Some results on global stability of a predator-prey system, J. Math. Biol. 12, 115-126 (1981). MR0631003 (83c:34069)

[3] H. I. Freedman, Deterministic Mathematical Models in Population Ecology, Marcel Dekker, New York, 1980. MR0586941 (83h:92043)

[4] G. F. Gause, The Struggle for Existence, Williams and Wilkins, Baltimore, 1934.

[5] C. S. Holling, The components of predation as revealed by a study of small-mammal predation of the European pine sawfly, Canad. Entomol. 91, 293-320 (1959).

[6] S.-B. Hsu, On global stability of a predator-prey system, Math. Biosci. 39, 1-10 (1978). MR0472126 $(57: 11837)$

[7] V. S. Ivlev, Experimental Ecology of the Feeding of Fishes, Yale University Press, New Haven, CT, 1961.

[8] N. D. Kazarinoff and P. van den Driessche, A model predator-prey system with functional response, Math. Biosci. 39, 125-134 (1978). MR0475989 (57:15570)

[9] R. E. Kooij and A. Zegeling, A predator-prey model with Ivlev's functional response, J. Math. Anal. Appl. 198, 473-489 (1996). MR1376275 (96j:92033)

[10] R. E. Kooij and A. Zegeling, Qualitative properties of two-dimensional predator-prey systems, Nonlinear Anal. 29, 693-715 (1997). MR.1452753

[11] Y. Kuang, Nonuniqueness of limit cycles of Gause-type predator-prey systems, Appl. Anal. 29, 269-287 (1988). MR0959804 (89h:92047)

[12] Y. Kuang, Global stability of Gause-type predator-prey systems, J. Math. Biol. 28, 463-474 (1990). MR:1057049 (91g:92017)

[13] Y. Kuang and H. I. Freedman, Uniqueness of limit cycles in Gause-type models of predator-prey systems, Math. Biosci. 88, 67-84 (1988). MR0930003 (89g:92045) 
[14] R. M. May, Stability and Complexity in Model Ecosystems, Princeton University Press, Princeton, 1974.

[15] M. L. Rosenzweig and R. H. MacArthur, Graphical representation and stability conditions of predator-prey interactions, Am. Naturalist. 97, 209-223 (1963).

[16] J. Sugie, Two-parameter bifurcation in a predator-prey system of Ivlev type, J. Math. Anal. Appl. 217, 349-371 (1998). MR:1492094 (98m:92024)

[17] J. Sugie, Uniqueness of limit cycles in a predator-prey system with Holling-type functional response, Quart. Appl. Math. 58, 577-590 (2000). MR1770656 (2002a:92020)

[18] J. Sugie, Homoclinic orbits in generalized Liénard systems, J. Math. Anal. Appl. 309, 211-226 (2005). MR2154037(2006d:34101)

[19] J. Sugie and T. Hara, Existence and non-existence of homoclinic trajectories of the Liénard system, Discrete Contin. Dynam. Systems 2, 237-254 (1996). MR1382509(97a:34127)

[20] J. Sugie and M. Katayama, Global asymptotic stability of a predator-prey system of Holling type, Nonlinear Anal. 38, 105-121 (1999). MR.1693000 (2000j:92026)

[21] J. Sugie, R. Kohno and R. Miyazaki, On a predator-prey system of Holling type, Proc. Amer. Math. Soc. 125, 2041-2050 (1997). MR.1396998 (97m:92005)

[22] J. Sugie, K. Miyamoto and K. Morino, Absence of limit cycles of a predator-prey system with a sigmoid functional response, Appl. Math. Lett. 9, 85-90 (1996). MR.1415457 